\title{
Kastrationsresistentes Prostatakarzinom Optimale Länge der systemischen Therapie
}

In der täglichen Praxis stellt sich einerseits die Frage, welche Behandlung in der aktuellen Situation einzuleiten ist, aber andererseits auch, wann eine aktive Tumortherapie zu beenden ist und die palliative Betreuung mit der besten supportiven Behandlung eingeleitet werden sollte. Dr. Robert Tauber, Klinikum rechts der Isar der TU München, gab diesbezüglich beim Deutschen Krebskongress Hilfestellung für die Entscheidungsfindung beim metastasieren kastrationsresistenten Prostatakarzinom (mCRPC).

Eine wichtige Information in der Entscheidung, die systemische Therapie fortzuführen oder zu beenden, ist die Prognose des Patienten. Prognose-beeinflussende Faktoren sind, in Bezug auf den Patienten, das Alter, der Allgemeinzustand, Begleiterkrankungen, die Patientenadhärenz und die Versorgungssituation. An Krankheitsfaktoren müssen die Metastasenlast, die Progressionsgeschwindigkeit, die viszerale Metastasierung insbesondere der Leber sowie die Entdifferenzierung des Karzinoms, beispielsweise eine neuroendokrine Differenzierung, beachtet werden. Schließlich beeinflussen auch Therapiefaktoren die Prognose. Dazu gehören die Anzahl und Art der Vortherapien sowie die Dauer des Ansprechens auf die Vortherapien.

Für Patienten in der metastasierten kastrationsresistenten Situation gibt es keine kurative Therapie, konstatierte Tauber, das muss dem Patienten auch kommuniziert werden. An palliativen Therapieoptionen, welche das Überleben verlängern können, stehen die AR-gerich- teten Medikationen, Chemotherapien sowie radioaktive Substanzen zur Verfügung. Die großen Zulassungsstudien für alle diese Substanzen zeigen allerdings in den Subgruppenanalysen keinen signifikanten Überlebensvorteil bei Patienten mit schlechtem Allgemeinzustand oder Viszeralmetastasierung. Auch die Wirkungs-Nebenwirkungsrelation trägt zur Indikationsstellung von palliativen Tumortherapien bei. Bei Patienten mit schlechter Prognose aufgrund von schlechtem Allgemeinzustand und viszeralen Metastasen ist die Beendigung der Systemtherapie daher wahrscheinlich im Sinne des Patienten.

Das Ziel der palliativmedizinischen Versorgung ist die Verbesserung der Lebensqualität durch eine effektive Behandlung von belastenden Symptomen. Häufige Symptome bei Patienten mit fortgeschrittenem Prostatakarzinom sind Schmerzen, Fatigue, Gewichtsverlust, Angst, Depression und organbezogene Symptome, wie beispielsweise Lähmungserscheinungen und Harnverhalt. Die Möglichkei- ten der Palliativtherapie sollten mit dem Patienten und seinen Angehörigen umfassend und frühzeitig besprochen werden, erinnerte Tauber. Bei Vorliegen von Knochenmetastasen ist beispielsweise laut interdisziplinärer S3-Leitlinie die lokale perkutane Bestrahlung bei persistierenden Knochenschmerzen, bei drohender spinaler Kompression, nach operativer Stabilisierung und bei erhöhtem Frakturrisiko empfohlen. Zudem soll eine osteoprotektive Therapie eingesetzt werden. Letztere ist wahrscheinlich nicht überlebensverlängernd und keine Prävention für Knochenmetastasen, kann aber skelettale Ereignisse verhindern oder verzögern.

Bei der Indikationsstellung von Tumortherapien komme es nicht darauf an, dem Leben mehr Jahre zu geben, sondern den Jahren mehr Leben zu geben, zitierte Tauber Cicely Saunders, die Begründerin der modernen Palliativmedizin. Die Entscheidungsfindung solle Informationen über alle Behandlungsmethoden beinhalten, die Patientensituation und Prognosefaktoren berücksichtigen, die Erstellung eines Behandlungsplans unter Berücksichtigung der persönlichen Präferenzen des Patienten umfassen und Informationen über alle verfügbaren Betreuungsangebote, wie Selbsthilfegruppen, Psychoonkologie, ambulante Pflege, stationäre Pflege und Hospize, geben.

Dr. Ine Schmale, Westerburg

Quelle: AUO-Symposium „Grenzen und Chancen der Therapie beim Prostatakarzinom" im Rahmen des Deutschen Krebskongresses (DKK) am 21. Februar 2018, Berlin. 